UCRL-JC-105560

PREPRINT

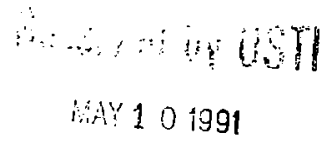

\title{
Emittance Measurement in a Magnetic Field
}

\author{
J. K. Boyd
}

This paper was prepared for submittal to the 1991 IEEE Particle Accelerafor Conference May 6-9, 1991, San Francisco, CA

April 15, 1991

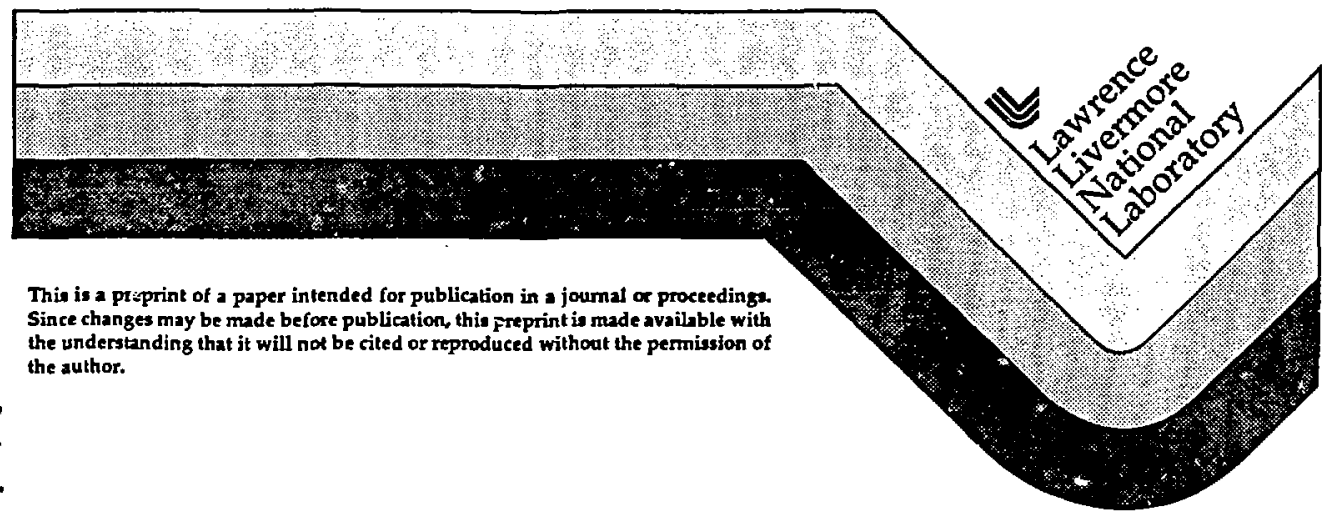




\section{DISCLAIMIER}

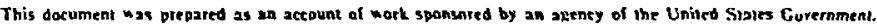
Yeilher the Linited States Corernment nor the University of Californis nor any at their emplojecs, makes any watranty. erpeess or implied of assumen sey legal liability or resonsibility for the aceuracy. compleseness. of usefulness of any infocmatioa, apparalus, product, or prucess dixclosed, or represents that ixs use would not infringe privately onned richts. Relerence herein to any specific commercial products, procesh or servite by tradt name. teademark, mandacturer, or othefw ice, does aot mecessarily constitufe or imply irs endorsement. recommendation. or iavoring by the United States Goverament of the University of California. The viens and opinions of authors expressed herein do not necesurily state or reflect those of the United States Governm : or the University of California, and shall nos be used for advertising or product endorsement purposes. 


\title{
EMITTANCE MEASUREMENT IN A MAGNETIC FIELD *
}

\author{
J. K. Boyd \\ Lawrence Livermore National Laboratory, University of California \\ Livermore, California 94550
}

\section{Abstract}

Emittance can be measured by intercepting an electron beam on a range thick plate and then observing the expansion of beamlets transinitted through small holes. The hole size is selected to minimize space charge effects. In the presence of a magnetic field the beamlets have a spiral trajectory and the usual field free formulation must be modified. To interpret emittance in the presence of a magnetic field an envelope equation is derived in the appropriate rotating frame.

\section{Introduction}

Emittance in a magnetic field can be measured by using an emittance mask or "pepper" pot plate to cetermine the change in radius of a small radius beamlet which transmits through the emittance mask. There is a straightforward envelope equation formula that is commonly used in experimenis with a beamlet in a field free region to determine emittance. However, in a magnetic field a beamlet does not travel in a straight line, but rather rotates at the cyclotron frequency in a spiral path. In order to analyze emittance measuring experiments, when a magnetic field is present it is necessary to examine the envelope equation in a frame which eliminates the effect of the spiral motior. To accomplish this goal the beamlet is described by an envelope equation applicable to a frame rotating at the cyclotron frequency.

For comparison we first derive the formula based on the field free emittance measurement. Next the envelope equation for a beamlet in a magnetic field is derived, introducing a new quantity $Q_{\theta}$, which enters the envelope equation quadratically with the emittance. To augment the interpretation of experimental data the envelope equation in the rotating frame is written with quantities expressed in terms of lab frame equivalents. $A$ discussion is given of the assumptions necessary to allow an identification of the $Q_{\theta}$ function with che familiar canonical angular momentun.

\section{Field Free Emittance Measurement}

The field free emittance is measured by observing the initial and finai radius of a beamlet which passes through a hole in a range thick emittance mask. Over a known distance the change in radius is related to the emittance by the envelope equation. For constant energy and assuming canonical angular momentum is zero, the rms envelope

* Work performed under the auspices of the U.S. Dept. of Energy by Lawrence Livermore Nationa Lab. under contract W-7405-ENG-48. equation [1] for current $I_{b}$ is,

$$
\frac{d^{2} R}{d z^{2}}-\frac{I_{b}}{17000 \gamma^{3} R}+\frac{1}{4} k_{c}^{2} R-\frac{E_{\text {beam }}^{2}}{\gamma^{2} R^{3}}=0
$$

Generally the beamlet transmits through a hole in the emittance mask small enough so the dynamics are domin nated by emittance rather than space charge. The criteria for an emittance dominated beam is that $I_{3} / 17000 \gamma<<$ $E_{\text {beam }}^{2} / R^{2}$, which for known current, $\gamma$ and expected emittance specifies a bound on the emittance mask hole radius. To analyze the beamlet it is assumed current is small enough to satisfy this criteria, and in a field free region $k_{c}=0$ so the governing equation is,

$$
\frac{d^{2} R_{\text {blet }}}{d z^{2}}-\frac{E_{\text {blet }}^{2}}{\gamma^{2} R_{\text {blet }}^{3}}=0
$$

where $R_{\mathrm{t} \text { let }}$ is the rms radius of the beamlet and $E_{\mathrm{blet}}$ is the emittance of the beamlet. Solving Eq.(2) the rms radius is then related to the emittance by,

$$
\sqrt{K_{1} R_{\text {blet }}^{2}-\frac{E_{\text {blet }}^{2}}{\gamma^{2}}}=K_{1} z+\sqrt{K_{1} R_{0}^{2}-\frac{E_{\text {blet }}^{2}}{\gamma^{2}}}
$$

where the constant $K_{1}=\left(d R_{\text {blet }} / d z\right)_{0}^{2}+E_{\text {blet }}^{2} /\left(\gamma R_{0}\right)^{2}$ depends on the initial conditions. In a typical experiment $\left(d R_{\text {ble: }} / d z\right)_{0}=0$ and we obtain,

$$
E_{\text {blet }}=\frac{\gamma R_{0}}{2} \sqrt{R_{\text {blet }}^{2}-R_{0}^{2}}
$$

For a known emittance mask hole size $\left(\sqrt{2} R_{0}\right)$ and measured final beamlet rms radius, the normalized rms emittance, $E_{\text {blet }}$ is given by Eq.(4). Under the assumption of a uniform phase space fill $I_{\text {blet }} / E_{\text {blet }}^{2}=I_{\text {beem }} / E_{\text {beam }}^{2}$ and the beam emittance can be expressed in terms of the currents and beamlet emittance,

$$
E_{\text {beam }}=\frac{\gamma R_{0}}{z} \sqrt{R_{\text {blet }}^{2}-R_{0}^{2}} \sqrt{\frac{I_{\text {beam }}}{I_{\text {blet }}}}
$$

Alternatively a number of beamlets may be used to obtain velocity angles at several beam radii and a phase space plot can then be constructed. The emittance is derived from the area of the circumscribing phase space ellipse.

\section{Enittance Measurement in a Magnetic Field}

In a magnetic field it is necessary to derive an envelope equation in a frame rotating with the beam. Generally for an experiment in which the goal is to achieve as small a beam focus as possible the initial beam emission is set up to guarantee the canonical angular momentum of the beam is zero. When the canonical angular momentum of the beam is zero the appropriate rotation frequency of the

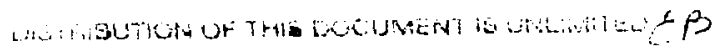


frame rotating with the beamlet is the cyclotron frequency. The starting point of the derivation is Eq.(17) from reference [1], where energy is assumed to be constant and self forces are neglected.

$$
\frac{d}{d t}\left(\frac{d \vec{r}}{d t}\right)=-\omega_{c} \hat{z} \times \frac{d \vec{r}}{d t}-\frac{1}{2 \gamma} \frac{d}{d t}\left(\gamma \omega_{c}\right) \bar{z} \times \vec{r}
$$

Neglecting self forces is a similar assumption to the no space charge criteria discussed previously. In Eq.(B) the cyclotron frequency contains the sign of the charge, $\omega_{e}=$ $q B_{2} / \gamma m c$. The desired rotating frame rotates in the same direction as an electron. For $B_{x}>0$ and $v_{z}>0$ an electron rotates in the $\hat{\theta}$ direction, so the correct angular rotation vector is $\left|\omega_{c}\right| \hat{z}$, and because for an electron the charge is negative, this is $-\omega_{c} \hat{z}$. Denoting by an asterisk the time derivative in the rotating frame, the relation between the time derivative in the fixed frame to that in the rotating frame is $d / d t=d / d t^{*}-\omega_{c} \bar{z} \times$. Using this relation in Eq.(6) gives,

$$
\frac{d}{d t^{*}}\left(\frac{d \vec{r}}{d t^{*}}\right)=\frac{d \omega_{c}}{d t^{*}} \hat{z} \times \vec{r}+\omega_{c} \hat{z} \times \frac{d \vec{r}}{d t^{*}}-\frac{1}{2 \gamma} \frac{d}{d t^{*}}\left(\gamma \omega_{c}\right) \hat{z} \times \dot{r}^{*}
$$

To get a force equation for the vector from the beamlet. centroid to the particle, $\vec{r}$, set $\vec{r}=\overrightarrow{r^{\prime}}+\vec{r}_{\text {rot }}$ where $\vec{r}_{\text {rot }}$ is the vector from the original origin to the center of rotation and the $\overrightarrow{\boldsymbol{r}^{\prime}}$ is the vector from the center of rotation to the particle. The beamlet centroid is assumed to be located by $\vec{h}$ from the original origin, and $\vec{h}=\vec{r}_{\text {ros }}+\vec{r}_{\mathrm{c}}$, where $\vec{r}_{c}$ locates the beamlet center from the center of rotation. The equation for $\vec{h}$ is the same as that of $\vec{r}$ in Eq.(7). Consequently $\vec{r}-\vec{h}$ must also satisfy the same equation and this gives the equation for $\overrightarrow{r^{\prime}}-\vec{r}_{c}=\vec{r}$ which yields the desired equation for $\vec{r}$.

$$
\frac{d}{d t^{*}}\left(\frac{d \vec{r}^{*}}{d t^{*}}\right)=\frac{1}{2} \frac{d \omega_{c}}{d t^{*}} \hat{z} \times \overrightarrow{r^{*}}+\omega_{c} \hat{z} \times \frac{d \overrightarrow{r^{*}}}{d t^{*}}
$$

To derive an envelope equation from Eq.(8) three subsidiary equations are obtained from dot products. An energy equation is obtained from the dot product with $d \vec{r} / d t$,

$$
\frac{d}{d t^{*}}\left(\frac{d \vec{r}}{d t^{*}}\right)^{2}=\frac{d \omega_{c}}{d t^{*}} l
$$

where $I=\left[\vec{r} \times d \vec{r} / d t^{*}\right] \cdot \hat{z}$. A virial equation is obtained from a dot product with $\vec{p}$,

$$
\frac{d^{2}}{d t^{-2}}\left(\frac{r^{-2}}{2}\right)-\left(\frac{d \vec{r}}{d t^{*}}\right)^{2}=-w_{c} l
$$

The third equation is obtained from the 2 component of $\vec{r} \times$ Eq. (8),

$$
\frac{d l}{d t^{*}}=\frac{r^{-2}}{2} \frac{d \omega_{c}}{d t^{*}}+\frac{\omega_{c}}{2} \frac{d r^{-2}}{d t^{*}}
$$

The average, $<>$ over a slice of the beamlet is defined to be a summation of a particle quantity over the radial slice, $<>\equiv \frac{1}{N} \sum_{i=1}^{N}$. For example $L=\langle l\rangle, R^{2}=\left\langle r^{* 2}\right\rangle$, and $V^{2}=\left\langle\left(d r^{*} / d t^{*}\right)^{2}\right\rangle$. Averaging over Eq.(9) gives,

$$
\frac{d}{d t^{*}} V^{2}=L \frac{d \omega_{c}}{d t^{*}}
$$

averaging over Eq.(10) gives,

$$
\frac{d^{2}}{d t^{-2}}\left(\frac{R^{-2}}{2}\right)=V^{2}-\omega_{c} L
$$

and averaging over Eq.(11) gives,

$$
\frac{d L}{d t^{*}}=\frac{R^{-2}}{2} \frac{d \omega_{c}}{d t^{*}}+\frac{\omega_{c}}{2} \frac{d R^{* 2}}{d t^{*}}
$$

From Eq.(14) it. can be shown $d Q_{\theta} / d t^{*}=0$ where $Q_{\theta}=$ $\gamma\left(L-\omega_{e} R^{2} / 2\right) / c$. Using Eq.(13) in Eq.(12) to eliminate $V^{2}$

$$
\frac{d}{d t^{*}}\left(R^{\cdot 3} \frac{d^{2} R^{*}}{d t^{-2}}+\frac{1}{4} \omega_{c}^{2} R^{* 4}\right)=0
$$

An integration of $\mathrm{Eq}$ (15) gives the beamlet envelope equation in the rotating frame,

$$
\frac{d^{2} R_{\text {blet }}}{d z^{2}}+\frac{1}{4} k_{\varepsilon}^{2} R_{\text {blet }}-\frac{E_{\text {blet }}^{2}+Q_{\theta}^{2}}{\gamma^{2} R_{\text {blet }}^{3}}=0
$$

where $E_{\text {blet }}^{2}=\gamma^{2} R_{\text {blet }}^{2}\left(V^{2}-\left(d R_{\text {blet }} / d t^{*}\right)^{2}-\left(L / R_{\text {blet }}\right)^{2}\right) / c^{2}$. Note that $E_{\text {blet }}$ and $L$ refer to the emittance and angular momentum of the beamlet. If rotation shear in the original beam which intercepts the emittance mask can be ignored and we assume $L \approx 0$ this leads to $Q_{\theta} \approx-\gamma k_{e} R^{2} / 2$ and then $\mathrm{Eq} .(16)$ becomes,

$$
\frac{d^{2} R_{\text {blet }}}{l z^{2}}=\frac{E_{\text {blet }}^{2}}{\gamma^{2} R_{\text {blet }}^{3}}
$$

Under this condition Eq.(5) cas be used to determine ernittance, and the answer is identical to the field free case.

For the most general situation the rotating frame quantities $E_{\mathrm{b} \text { et }}^{2}$ and $Q_{\theta}^{2}$ need to be related to the stationary lab frame. $E_{b l e t}^{2}$ is a preserved quantity since there is no scattering and $Q_{\theta}^{2}$ is preserved as a consequence of Eq.(14). It is therefore only necessary to find the relationship between the lab and rotating frame quantities at the creation position of the beamlet and it is then known for all positions. From the usual definitions $V^{2}=V_{\text {lab }}^{2}+\omega_{c}^{2} R_{\text {blet }}^{2}$ and also $L=L_{\mathrm{lab}}+\omega_{c} \Omega_{\mathrm{blet}}^{2}$. Tlus the definition of the beamlet emittance can be expressed in terms of lab quantities at the creation position of the beamlet,

$$
E_{\text {blet }}^{2}=\gamma^{2} R_{\text {blet }}^{2}\left(V_{\text {lab }}^{2}-\left(d R_{\text {blet }} / d t^{*}\right)^{2}-\left(L_{\text {lab }} / R_{\text {blet }}\right)^{2}\right) / c^{2}
$$

and the rotating frame quantity $Q_{\theta}$ is expressed in terms of lab angular momentum,

$$
Q_{\theta}=\frac{\gamma}{c}\left(L_{\mathrm{lab}}+\frac{1}{2} \omega_{\mathrm{c}} R_{\mathrm{blet}}^{2}\right)
$$

The expression on the right hand side of Eq.(19) is very similar to the definition of the whole beam canonical angular momentum. The correspondence is however incomplete 
since the whole beam canonical angular momentum is an average over all particles and $L_{\text {lab }}$ has only included particles contained in the beamlet. To make further progress it is assumed that any part of the whole beam is characterized by any other smaller subset. When this assumption is satisfied we have $Q_{\theta}=P_{\theta}$.

In Eq.(18) there is an additional problem beyond the question of uniformity because $d R_{\text {blet }} / d t^{*}$ is the convergence or divergence of the beamlet with respect to its center, whereas the analogous quantity for the whole beam is with respect to the whole beam center. This means in general the beamlet quantity particles bave a net inward or outward motion with respect to the whole beam center. For the beamlet this is like a steering kick. To avoid this problem it is assumed the whole heam arrives at the beamlet creation location at a waist. At this location we then have, from the assumption of uniform phase space fill,

$$
\begin{aligned}
E_{\text {blet }}^{2} & =\frac{I_{\text {blet }}}{I_{\text {beam }}} E_{\text {beam }}^{2} \\
& =\mathcal{X}^{\prime} E_{\text {beam }}^{2}
\end{aligned}
$$

The $\mathcal{X}$ function can be expressed in terms of the beam $a_{\text {beam }}$ and beamlet radius $a_{\text {blet. }}$. The beanlet current density is assumed to be uniform over its area with a value given by the whole beam (uniform phase space) current density at the beamlet centroid position $r_{h}$.

$$
J_{\text {blet }}=\frac{2 I_{\text {beam }}}{\pi a_{\text {beam }}^{2}}\left[1-\left(\frac{r_{h}}{a_{\text {beam }}}\right)^{2}\right]
$$

Using $I_{\text {blet }}=\pi a_{\text {blez }}^{2} J_{\text {blet }}$ and Eq.(21) we find, $\mathcal{X}=2\left(a_{\text {blet }} / a_{\text {beam }}\right)^{2}\left(1-\left(r_{h} / a_{\text {beam }}\right)^{2}\right)$. The canonical angular momentum of the whole beam is assumed to be zero and then the beamlet envelope equation in the rotating frame is,

$$
\frac{d^{2} R_{\text {blet }}}{d z^{2}}+\frac{1}{4} k_{r}^{2} R_{\text {blet }}-\frac{\lambda^{\prime} E_{\text {beam }}^{2}}{\gamma^{2} R_{\text {blet }}^{3}}=0
$$

From Eq.(22) the radial spread of a beamlet can be used to determine $E_{\text {beam }}$.

Often in experiments an attempt is made to eliminate the field in the beamlet expansion region. Thus an interesting comparison is the size of the second and third terms in Eq.(22). This comparison indicates how successful one must be experimentally at eliminating field leakage so the emitt,ance dominates the expansion of the beamlet. The two terms are equal at a field strengtl of,

$$
B \text { [gauss] }=\frac{3400 E_{\text {beam }} \sqrt{. \mathfrak{t}}}{R_{\text {blet }}^{2}}
$$

The condition given by Eq.(23) specifies the field such that the beamlet does not cliange radius if the initial beamlet $d R / d z=0$. For $B$ less than the value given hy Eq.(23) the emittance is dominant, and when it is greater the magnetic field is dominant. For nominal values of $E_{\text {beam }}=0.25$ rad-cm, $R_{\text {blet }}=0.1 \mathrm{~cm}$ and $\boldsymbol{X}=0.01 \mathrm{~cm}$ Eq.(23) gives $B=8500$ gauss.
In a situation where $k_{\varepsilon}$ is constant in $z \mathrm{Eq} .(22)$ can be solved,

$$
\begin{aligned}
k_{\mathrm{c}} z= & \sin ^{-1}\left[\frac{k_{c}^{2} R_{\mathrm{blet}}^{2}-2 K_{2}}{\sqrt{K_{3}}}\right] \\
& -\sin ^{-1}\left[\frac{k_{c}^{2}\left(R_{\mathrm{blet}}^{0}\right)^{2}-2 K_{2}}{\sqrt{K_{3}}}\right]
\end{aligned}
$$

where the constant $K_{3}=\left(d R_{\text {blet }}^{0} / d z\right)^{2}+0.25 k_{c}^{2}\left(R_{\text {blet }}^{0}\right)^{2}+$ $\left(\mathcal{H} E_{\text {beam }}^{2}\right) /\left(\gamma R_{\text {blet }}^{0}\right)^{2}$, is from the initial conditions, and $K_{3}=4 K_{2}^{2}-4 \mathcal{X}^{2} E_{\text {beam }}^{2}\left(k_{c} / \gamma\right)^{2}$. Since $E_{\text {beam }}$ is contained in the definition of $K_{2}$ and $K_{3}$, it can be seen that Eq.(24) is a transcendental equation for $E_{\text {beam. When }} k_{c}$ is small the starting point for a numerical solution is to use the field free solution for $E_{\text {beamn }}$.

In an experiment where emittance is known Eq.(22) can be solved to give the bearilet radius at a particular location.

$$
\begin{aligned}
R_{\text {blet }} & =\frac{1}{k_{c}} \sqrt{2 K_{2}+\sqrt{K_{3}} \sin \left(k_{\mathrm{e}} z+\sin ^{-1}[\varphi]\right)} \\
\varphi & =\frac{k_{c}^{2}\left(R_{\text {blet }}^{0}\right)^{2}-2 K_{2}}{\sqrt{K_{3}}}
\end{aligned}
$$

As a test of the derivation of Eq.(22) and the Eq.(25) solution, a particle code was used to follow a beamlet over a distance of $71.2 \mathrm{~cm}$ with 1600 partices. The magnetic field was 2550 gauss, $a_{\text {beam }}=1 \mathrm{cml}$, $a_{\text {blet }}=0.15 \mathrm{~cm}$, $E_{\text {beam }}=0.25$ tad-cm, $\gamma=34$ and $R_{\text {blet }}^{0}=0.104$. The Eq. (25) solution gives $R_{\text {blet }}=0.48 \mathrm{~cm}$ for these parameters and the computer simulation gives a value of $R_{\mathrm{blet}}=0.50$ cm which agrees to $4 \%$.

\section{Summary}

The formula relating the emittance to the initial and final t.ransmitted heamlet radius has been derived for a field free measurement. A beamlet envelope equation ieglecting space charge and assuming constant energy has been derived in a frame rotating at the cyclotron frequency. It was found when the beamlet does not have any angular momentum the magnetic field term in the rotating frame envelope equation is canceled by the $Q_{\theta}$ contribution. In this situation the rotating frame envelope equation is identical to the field free case and the original relation for emittance in terms of initial and final transmitted beamlet radius is applicable. In the general case an equivalence between lab canonical angular momenturn and $Q_{\theta}$ has been shown and the assumptions necessary for this to be valid have heen discussed. For uniform phase space the envelope equation in the rotaing frame was written in terins of the lab or whole beam emittance. In order to do this it was necessary to assume a beam waist at the beamlet creation position. It is the measurentent of the whole beam enittance that is the usual experimental objective. An expression that may be numerically solved has been determined to solve for the whole beam emittance, given experimental values of beam energy, magnetic field, initial and final beanlet radius. For a situation where emittance is known a solution lias been derived for the beamlet radius at any position. This solution has been tested against a particle code and found to agree to within $4 \%$.

References

[1] E.P.Lee, R.K.Cooper, Part. Acc.,7, p.83. (1976) 